\title{
Los rankings académicos y la distribución por género de las universidades
}

\author{
Teodoro Luque-Martínez*; Nina Faraoni* y Luis Doña-Toledo* \\ *Universidad de Granada, Dpto. Comercialización e Investigación de Mercado. \\ Facultad de Ciencias Económicas y Empresariales \\ Correo-e: tluque@ugr.es | ORCID iD: https://orcid.org/0000-0003-1282-6822 \\ Correo-e: ninaf@ugr.es | ORCID iD: https://orcid.org/0000-0003-1582-5104 \\ Correo-e: luisdt@ugr.es | ORCID iD: https://orcid.org/0000-0003-2238-2671
}

Recibido: 13-03-2019; 2a versión: 15-07-2019; Aceptado: 15-07-2019.

Cómo citar este artículo/Citation: Luque-Martínez, T.; Faraoni, N.; Doña-Toledo, L. (2020). Los rankings académicos y la distribución por género de las universidades. Revista Española de Documentación Científica, 43 (2), e261. https://doi. org/10.3989/redc.2020.2.1663

Resumen: Este trabajo examina la distribución por género de las universidades, a través de un porcentaje de mujeres dentro del alumnado, y comprueba si tal distribución tiene alguna relación con la posición de las universidades en los rankings universitarios. Se han seleccionado siete rankings, globales y sintéticos, disponiendo de datos de la distribución por género del alumnado para 924 universidades. Los resultados confirman que las universidades con una equilibrada distribución entre hombres y mujeres presentan mayores puntuaciones, por tanto, mejores posiciones en los rankings. Las universidades con mayor presencia femenina en el alumnado son las de ratio más alto de estudiantes por profesor y las que alcanzan mayores valoraciones medias en los indicadores de internacionalización. En los indicadores de premios Nobel y medallas Fields, e ingresos procedentes de la industria, las universidades con mayor presencia masculina tienen mayores puntuaciones medias. Los resultados pueden contribuir a la mejora de las políticas de igualdad en las universidades, así como a orientar las decisiones estratégicas y de gestión de las mismas.

Palabras clave: Rankings universitarios; distribución por género y universidad; igualdad de género.

\section{Academic rankings and the distribution by gender of the universities}

Abstract: This paper examines the distribution of the universities by gender through a percentage of women within the student body, and checks if such distribution has any relation to the position of the universities in the university rankings. Seven rankings, have been selected, both global and synthetic, and a joint database collecting all data on gender distribution of students from 924 universities was created. The results confirm that universities with a more balanced distribution between men and women receive higher scores and, therefore, better ranking positions. The universities with the highest female presence in the students are those with the highest ratio of students per teacher and those with the highest average scores in internationalization indicators. In the indicators of Nobel prizes, Fields medals, and income from the industry, those universities with the highest male presence have higher average scores. These results can contribute to the improvement of equality policies in universities, as well as to guide the strategic and management decisions.

Keywords: University rankings; composition by sex and university; gender equality.

Copyright: (c) 2020 CSIC. Este es un artículo de acceso abierto distribuido bajo los términos de la licencia de uso y distribución Creative Commons Reconocimiento 4.0 Internacional (CC BY 4.0). 


\section{INTRODUCCIÓN}

En la conferencia "Going Global" del British Council, celebrada en 2013 en Dubái, una agrupación internacional de mujeres no solamente pidió que la igualdad entrara a formar parte de los indicadores utilizados para la medición de los resultados de las universidades, sino que se convirtiera en un elemento clave para medir el desempeño de la calidad de las instituciones de educación superior. Esto conduce a la pregunta de si la mayor presencia de mujeres (o de hombres) en la universidad tiene alguna relación con su posición en los rankings internacionales.

Se han necesitado casi 800 años para que la presencia de mujeres y hombres en las universidades se equilibre, y no en todos los sitios. La primera universidad fundada (Universidad de Bolonia) prohibía el ingreso de las mujeres. En el año 1400, se empezó a admitir su matriculación en casos excepcionales (Caputo y otros, 2016). A principio del siglo $X X$, ni un $1 \%$ del total del alumnado eran mujeres (Giménez-Salinas, 2005) un siglo después son mayoría en muchas universidades (UNESCO, 2018).

El retraso de la incorporación de la mujer a la universidad, en la misma proporción que el hombre, tiene que ver con los roles asignados a ambos. La literatura identifica varias razones. Primero, la «liberación» de mano de obra del trabajo doméstico. Hasta bien entrada la segunda mitad del siglo $X X$, el hecho de que una hija quisiese estudiar era quebranto para la familia, porque dejaba de ayudar en el hogar y era una pérdida para la economía doméstica. También los cambios en las actividades domésticas como consecuencia de innovaciones (agua corriente, electricidad, etc.) liberaron a las mujeres de carga de trabajo, haciendo que los costes de oportunidad de estudiar para la familia resultaran más interesantes (Martínez García, 2007). Además, no se consideraba necesaria una educación formal para las mujeres, puesto que las funciones que se le asignaban eran cuidar de los hijos y cuidar del hogar (Carmona-Valdés, 2015).

El cambio se dio con la expansión del sector servicios de la economía, que incrementó los empleos del sector de media cualificación (Shavit y Blossfeld, 1996). En el nuevo contexto socioeconómico, se hizo cada vez más habitual y necesario el sueldo de ambos cónyuges para mantener una familia (Alba, 2000). Así aumentó la participación laboral femenina y la necesidad, a la vez que oportunidad, de una mayor preparación. En todo este proceso, tiene mucho que ver la propia evolución social, junto con el interés creciente de la necesidad de igualdad en una institución pública como la universidad, que es un reflejo de la sociedad en la que se inserta.
En este contexto, el objetivo del presente trabajo es analizar la presencia femenina dentro del alumnado en una institución socialmente tan importante como es la universidad, y conocer las implicaciones que tiene. Es decir, comprobar si dicha presencia tiene alguna relación con la posición de las universidades en los rankings globales, y con los indicadores que se utilizan para confeccionar tales clasificaciones. Se trata de responder si una mayor, menor o equilibrada presencia de mujeres entre los estudiantes es determinante a la hora de que las universidades tengan posiciones mejores o peores, o si eso genera diferencia en los indicadores con los que se elaboran los rankings.

La literatura no ha tratado este asunto, no hay referencias o estudios previos que aborden esta temática, ni que respondan a estas cuestiones, además de ser complicado de medir todo lo relacionado con la igualdad de género en las universidades (a tal propósito, se señala que el Center for Science \& Technology Studies (CWTS) de la Universidad de Leiden, que se ocupa de la creación del ranking Leiden, hace poco introdujo, entre sus indicadores, uno relativo al género, en particular a la ratio de publicaciones hombres/mujeres, disponible para 963 universidades (Sugimoto y Larivière, 2019; Wagner, 2019). Por ello, se analiza la presencia de la mujer en el alumnado en las principales universidades del mundo para comprobar si en función de ello se presentan diferencias en los indicadores de desempeño. Para ello se trabaja con más de novecientas universidades de las que se dispone de información sobre su distribución por género y con siete rankings universitarios globales, utilizando un indicador de porcentaje mujeres/hombres.

\section{UNIVERSIDAD: ROL Y ACCESO DE LA MUJER}

Todo lo relacionado con la igualdad de género en la universidad ha llegado a ser un importante tema en la actualidad (Wagner, 2019). En los últimos años, los movimientos para los derechos de las mujeres han proliferado y han despertado el interés por un asunto que, hasta hace poco, no generaba polémica en una sociedad regida por estructuras arraigadas, que veían el rol de la mujer orientado al cuidado de la casa y de la familia (Tzanakou y Pearce, 2019). Las consecuencias de estas estructuras sociales siguen reflejándose a día de hoy, y las acciones que se cumplen en el contexto académico son colectivas, sistemáticas y normalizadas, yendo más allá del comportamiento individual de cada uno (Wagner, 2019). Por lo general, el aumento de la presencia de mujeres en la educación ha reducido, pero no eliminado, la discriminación por género como se pone de relieve en distintos ámbitos de la vida académica. 
Por un lado, con referencia a las estudiantes, esto se manifiesta en la elección de estudios universitarios. Es frecuente encontrar referencias que demuestran que la presencia de mujeres se concentra en estudios considerados propiamente "femeninos" por los roles tradicionalmente atribuidos a la mujer (Duart y otros, 1996; Gámez y Marrero, 2003; Papadópulos y Radakovich, 2003; Sáinz y otros, 2004). Los hombres suelen elegir profesiones relacionadas con los estudios técnicos, mientras que las mujeres se decantan por los estudios sociales y humanitarios (Zeegers, 2004). Hay titulaciones con un tradicional predominio femenino como son psicología, enfermería, historia, pedagogía, educación social y magisterio. Por el contrario, las hay predominantemente masculinas como las ingenierías, arquitecturas e informática (Sáinz y otros, 2004; Larivière y otros, 2013). El estado de opinión conduce a una menor propensión de ellas a elegir estudios universitarios relacionados con las TICs y la Alta Tecnología, hecho que puede influenciar la futura integración de las mismas en entornos profesionales relacionados con estos ámbitos. Con el tiempo, esto se traduce en la subrepresentación de las mujeres en el campo de la investigación científica, en particular en tecnología, técnica e ingeniería, donde las mujeres autoras suponen un porcentaje muy bajo del total de las publicaciones (Larivière y otros, 2013). La literatura muestra intentos para dar una respuesta al por qué de este fenómeno (Muñoz y Mullet, 1990; López-Sáez, 1995; Duart y otros, 1996; NavarroGuzmán y Casero-Martínez, 2012).

Algunos autores afirman que los motivos que subyacen a elecciones distintas son diferentes según el sexo: ellas eligen buscando la satisfacción de sus intereses o la preferencia personal, y ellos se guían por las oportunidades profesionales que les brindará la opción elegida (Muñoz y Mullet, 1990; Duart y otros, 1996; Luque-Martínez y otros, 2014, 2015). También se afirma que la única diferencia entre hombres y mujeres reside en el hecho de que los hombres tienen en cuenta la retribución futura que les permite la carrera que hayan decidido estudiar (López-Sáez, 1995). Tales resultados están en la misma línea de los resultados obtenidos por el estudio CIDE (1998), según el cual los hombres se guían por el hecho de ganar más dinero, y las mujeres por el consejo dado por sus padres y profesores. Por otro lado, según otros autores, las mujeres eligen la carrera más por criterios vocacionales, porque les gusta o por el deseo de ayudar a los demás, mientras que los hombres eligen más por criterios relativos a salidas profesionales o de retribución (Navarro-Guzmán y Casero-Martínez, 2012). A esto hay que añadir lo que Pérez-Tyteca y Castro (2011) denominan "an- siedad matemática", aludiendo a un estado ansioso y estresado relacionado con la matemática tanto en la vida académica como en la vida cotidiana. Según estos autores, existe una predisposición de las mujeres a padecer situaciones de incomodidad relacionadas con la ansiedad matemática, aunque es algo muy cuestionado en la actualidad. A día de hoy, no hay evidencia empírica de que las diferencias en la elección puedan deberse a diferencias intelectuales (Bird, 2011).

Por otro lado, una corriente en la literatura postula la existencia de un conjunto de creencias y valores de cada persona y del imaginario colectivo y cultural de cada sociedad que condiciona y normaliza el comportamiento de hombres y mujeres en rasgos percibidos como típicamente "masculino" y típicamente "femenino" (Carmona-Valdés, 2015). Por ejemplo, Trotter (2017) analiza la evaluación de la titulación de enfermería como típicamente femenina, por lo cual se está produciendo progresivamente una reestructuración de los recursos educativos y sociales en la sociedad. En el subconsciente de las personas, y también en el pensamiento colectivo, existen unas creencias de género, unas percepciones, tanto sobre nosotros mismos cuanto sobre los demás individuos. Según el estudio de Aiston (2011), la desigual posición de las mujeres en las instituciones académicas, comparada con la de los hombres, se debe a razones deontológicas comunes en la sociedad. Estas creencias de género sobre uno mismo, y sobre sus capacidades, pueden llegar a influenciar a los individuos hasta el punto de que lleguen a tomar decisiones en su vida diaria guiados por estas valoraciones personales erróneas.

De esta forma, también las decisiones relacionadas con las carreras profesionales pueden verse influenciadas por los roles de género y hacer que se tomen diferentes direcciones que, en ausencia de estas creencias de género, no hubiesen coincidido. Según Correll (2001), estas creencias acerca de las capacidades de hombres y mujeres suelen influenciar las vidas de las personas más temprano de lo que parece, y pueden estar relacionadas con las afirmaciones mencionadas anteriormente por Pérez-Tyteca y Castro (2011) acerca de la "ansiedad matemática".

Las influencias de los entornos crean percepciones inexactas y barreras percibidas. McWhirter (1997) afirma que existen barreras percibidas cuando se trata de lograr objetivos educativos y profesionales. Con respecto a sus futuros trabajos, las mujeres tienen más probabilidades de percibir la discriminación por motivos de sexo que los hombres. Estas percepciones no solo existen en ámbito laboral y entre el profesorado 
femenino, sino que existen desigualdades, por lo menos en la percepción del trato recibido por hombres y mujeres, también entre estudiantes de posgrado (Seagram y otros, 1998). Desde el punto de vista de la teoría de la identidad social, se puede concluir que la percepción de sí mismos de los individuos parece afectar tanto a las autoevaluaciones como a las evaluaciones externas (Lobel y Clair, 1992).

Hoy en día, es posible encontrar desigualdades por género entre el profesorado universitario $y$, en ciertos casos, entre el alumnado, a pesar de las medidas adoptadas por parte de las instituciones de educación superior (Timmers y otros, 2010; Larivière y otros, 2013; Teelken y Deem, 2013; Tzanakou y Pearce, 2019). Se han realizado esfuerzos para lograr una mayor equidad de género (Van den Brink y otros, 2010), por ejemplo, con el fomento del uso de lenguaje inclusivo o con la modificación de los libros de texto desde la infancia, para que los niños se acostumbren a derrumbar barreras ideológicas difíciles de abatir. Pero en la mayoría de los países, siguen existiendo construcciones sociales como el "think manager, o think male", que son argumentos para alcanzar mayores logros y metas (Powell y otros, 2002; Willemsen, 2002), aunque, por regla general, el rendimiento educativo de las mujeres es más elevado que el de los varones a lo largo de toda la enseñanza formal (Lozano, 2004; Luque-Martínez y otros, 2014, 2015).

Los obstáculos también se manifiestan finalizados los estudios, pues la inserción laboral muestra diferencias por género en cuanto a condiciones laborales o sueldo (García, 2014). Según Martínez García (2007) el salario medio de las mujeres es más bajo que el de los hombres, por eso, para ellas, el coste de oportunidad también es menor. Según Light y Ureta (1995), investigando el historial de trabajo, para los salarios de las mujeres se estimaba un crecimiento del $56 \%$ en 8 años de experiencia profesional, frente a un $82 \%$ de crecimiento de los hombres. En la actualidad, incluso se ha demostrado, que las mujeres tienen menores posibilidades de encontrar empleo por los contactos o las redes informales entre profesionales (Wright, 2016).

En suma, hay una discriminación ocupacional (Larivière y otros, 2013), cuyos efectos no disminuyeron con la llegada de la globalización (Gauchat et al, 2012). Las percepciones populares están convencidas de que estos obstáculos dependan de las características intelectuales de hombres y de mujeres $y$, por consecuencias, de las libres elecciones de cada uno (Igbaria y Wormley, 1992; Summers, 2005; Hoff Sommers,
2008). Además, la falta de reconocimiento de la existencia de barreras de género, por parte de los directivos de las universidades, sugiere la necesidad de una mayor difusión de los resultados de la investigación sobre cómo funcionan y por qué estas barreras perjudican desproporcionadamente a las mujeres (Bird, 2011).

En la educación superior, desde hace unos años, se ha empezado a debatir sobre el problema de la desigualdad, un buen ejemplo lo constituye el seminario "Absent Talent: Women in Research and Academic Leadership "(2012-2013), celebrado en Hong Hong, Tokyo y Dubai, que promueve el "Manifiesto for Change" que recoge seis propuestas para fomentar la presencia femenina en el ámbito universitario (Grove, 2013). En definitiva, aunque se haya avanzado hacia la igualdad en la investigación, la remuneración y la posibilidad de promoción laboral, es necesario seguir avanzando (Braidotti, 1994).

\section{LA PRESENCIA DE LA MUJER EN LA UNI- VERSIDAD}

La disposición de estadísticas sobre presencia de mujeres en la universidad, más aún en ámbito global, tradicionalmente ha sido insuficiente (Arranz, 2001). Recientemente, fuentes como el Global Gender Gap Report del World Economic Forum (2017), o los datos de las estadísticas UNESCO (2018), ofrecen información con más detalle.

Con respecto a la universidad, en más del $80 \%$ de los países, la mayoría de los titulados universitarios son mujeres. Según el informe estadístico 2018 del Ministerio de Ciencia, Innovación y Universidades, es una tendencia bastante recurrente la de encontrar más mujeres que hombres, sobre todo con referencia a los países occidentales. En España, el 54\% del alumnado son mujeres, tanto en nivel de Grado, como en Máster (MCIU, Datos y Cifras del Sistema Universitario Español, 2016). En Europa, la tendencia es la misma, pues la brecha de género en las admisiones universitarias en Reino Unido, en 2016, sube a un nivel récord (European Institute for Gender Equality, 2017) siendo 5,6 puntos mayor la presencia femenina.

Las áreas como las ingenierías, las técnicas, informática, tecnología, servicios y ciencias de la naturaleza son caracterizados por mayor presencia de hombres. Casi tres veces más mujeres que hombres eligen estudiar educación, salud y titulaciones sociales en los países de la Unión Europea, y esta tendencia no ha cambiado desde 2005 (European Institute for Gender Equality, 2017). 
Esto conduce a la necesidad de emprender medidas transformadoras que modifiquen la posición de las mujeres y el acceso a ciertas titulaciones (Ion y otros, 2013), puesto que hay autores que afirman que sería conveniente favorecer el acercamiento de las mujeres a la Alta Tecnología y a las TICS, beneficiando así la competitividad y el crecimiento económico del país (Mauleón y Bordons, 2014).

En relación al profesorado en el mundo, las maestras de escuela primaria suponen un $64,11 \%$ del total, mientras que, en secundaria, en 2015, las mujeres eran 17.559.420 (World Bank, 2016). En España, según el Ministerio de Educación (2016), las mujeres representan el $67,8 \%$ del total de profesores en primaria y el $55,6 \%$ en secundaria. En otros países como Reino Unido, las mujeres son $84 \%$ del total de profesores en primaria y el $56 \%$ en secundaria (Conley y Jenkins, 2011).

En la universidad, en la reflexión de Hernández Armenteros y Pérez García (2018), se señala la mayor presencia de mujeres en el total de contratados $(51,77 \%)$. El colectivo de profesores jóvenes es el que más mujeres tiene $(40,06 \%)$ con respecto a los otros colectivos. La mayoría de estas profesoras tienen menos de 40 años. Sin embargo, a medida que sube el cargo considerado, el porcentaje de presencia de mujeres disminuye, llegando casi al $25 \%$ del total para la categoría de los catedráticos. Este contexto laboral educativo se caracteriza por un sistema de participación en "pirámide" donde las mujeres dedicadas a la docencia se establecen en los niveles educativos más bajos (infantil, primaria y secundaria) (Díez Gutiérrez y otros, 2009).

Respecto a los puestos directivos, la presencia de mujeres tiene un importante déficit de liderazgo en las universidades. Pocas ocupan puestos de rectoras o vicerrectoras (Walby, 2011, Box-Steffensmeier et al. 2015; Howe-Walsh y Turnbull, 2016). Aunque esa brecha esté disminuyendo, el problema persiste en los cargos académicos más altos (Hernández Armenteros y Pérez García, 2018). Desde el punto de vista de la psicología se ha argumentado la falta de puestos directivos de mujeres en el ámbito universitario debido a los factores de "miedo al éxito", y las "prioridades vitales" (Grañeras, 2003; Bird, 2011) denominándose este fenómeno como "techo de cristal", fruto de la costumbre social y cultural de la mayoría de las sociedades (Grañeras, 2003). Este "techo de cristal" hace referencia a puestos de directivos ocupados por mujeres, donde existe una invisible barrera que restringe el adelanto de estos grupos a los más altos cargos ejecutivos (Morrison y otros, 1987; Igbaria y
Wormley, 1992; Stroh y otros, 1992; Greenhaus y Callanan, 1994; Matus-López y Gallego-Morón, 2015; Howe-Walsh y Turnbull, 2016).

Por último, en cuanto a la presencia en el ámbito de la investigación, las mujeres llegan a ser un tercio de los investigadores pues los hombres son un $71 \%$ de todos los investigadores (UNESCO, 2018). Con respecto al porcentaje de autoría de las investigaciones, las mujeres suponen alrededor de un $30 \%$ del total (Larivière y otros, 2013; Wagner, 2019), y sus publicaciones se encuentran especialmente concentradas en el campo de las ciencias sociales y de las humanidades (Sugimoto y Larivière, 2019).

Globalmente, el nivel de estudios finalizados de los empleados se distribuye de la siguiente forma: un nivel básico lo tiene el 31,2\% de los hombres frente a un $25,1 \%$ de las mujeres; un nivel intermedio (enseñanza secundaria) lo alcanzan el $35 \%$ de los hombres frente al $31,8 \%$ de las mujeres; y finalmente, las personas que obtienen un nivel alto de estudios (estudios universitarios) son por un $25,6 \%$ mujeres y por un $19,4 \%$ hombres (World Economic Forum, 2017). En España, las mujeres suponen el $38 \%$ de investigadoras en las universidades y sólo un $27 \%$ de mujeres directivas en el sector empresa (CE. Comisión Europea, 2009). Lo mismo pasa en el ámbito de la actividad tecnológica a nivel nacional, donde solamente un $29 \%$ de las patentes consideradas presentan al menos una mujer entre sus creadores. En el ámbito académico el $48 \%$ de las patentes presenta al menos una mujer entre sus creadores, mientras que en el ámbito de la empresa el dato se reduce a un $28 \%$ (World Intellectual Property Organization, WIPO, 2016).

Estos datos muestran que las mujeres sufren los dos tipos de discriminación detectados por Mauleón y Bordons (2014), y que ellas denominan como segregación. Por un lado, la discriminación horizontal que se presenta cuando la presencia femenina se agrupa en una sola área de estudio (como Humanidades y Ciencias Sociales) y, por otro lado, la discriminación vertical cuando cuanto más se ascienda en la jerarquía profesional, menor presencia de mujeres (menos mujeres catedráticas).

En definitiva, en los últimos 150 años las mujeres han podido acceder a la universidad con la misma libertad que los hombres, aunque se siguen apreciando importantes diferencias en cuanto a actividad investigadora y altos niveles jerárquicos, presencia en enseñanza técnicas y, sobre todo, número de estudiantes femeninas en ciertas áreas geográficas como Asia y América Latina (Tidball, 1976; Tzanakou y Pearce, 2019). 


\section{LA COMPOSICIÓN POR GÉNERO DE LAS UNIVERSIDADES Y LOS RANKINGS INTER- NACIONALES}

La incorporación progresiva de las mujeres en la universidad no ha ido siempre acompañada de normativas y procesos que hayan garantizado la igualdad (Morley, 2014), además, se conoce muy poco sobre el efecto del género en el éxito de los resultados, es decir, en qué manera afecta positivamente o negativamente la menor o mayor presencia de mujeres en las instituciones (Igbaria y Chidambaram, 1997).

Para poner en relación la presencia de la mujer, o la composición de la universidad según sexo, con el desempeño de las universidades hay que conocer, es decir, medir el desempeño de las instituciones mediante un conjunto de indicadores, además del grado de presencia de mujeres. Desde una perspectiva institucional, este propósito se puede lograr analizando los datos relativos a los rankings universitarios. Aunque estos rankings hayan sido objeto de muchas críticas (Docampo, 2008 y 2010; Hazelkorn, 2012; Luque-Martínez y Barrio-García, 2016), son herramientas útiles para una gran variedad de análisis. Hasta donde conocemos, no hay literatura que analice la relación entre la distribución universitaria por género y los indicadores de desempeño de las universidades, así como su posición en los rankings. En definitiva, se trata de ver la relación entre la distribución por género de las universidades con su producción científica (y otros indicadores bibliométricos), con su reputación (académica, de investigación o la que se tiene en el mercado laboral), con su grado de internacionalización, con su nivel de relación con la industria, o bien con la posición que ocupan las universidades en los rankings internacionales (Luque-Martínez y Faraoni, 2020). La cuestión es conocer la relación de estas diferencias constatadas con las posiciones que tienen las universidades en los rankings internacionales, y con los indicadores con los que se elaboran estos rankings.

Uno de estos indicadores, sin duda interesante para el estudio, es la reputación. La reputación es un constructo que está formado por varios elementos $y$, uno de estos elementos, incluye la inclinación ética de la institución hacia la inclusión y la igualdad de género. Con respecto al prestigio y a la reputación de las universidades, a menudo se hace referencia a ellos a través de elementos tradicionales como Premios Nobel, citas, recursos económicos, etc., pero poco se habla de cómo y en qué medida el entorno universitario, formado por los propios estudiantes, puede influenciar o estar relacionado con la creación de la idea de prestigio y de reputación (Mora, 2017).
Como lugar de generación y transmisión de conocimiento y valores, la universidad ocupa un lugar irremplazable como centro de creación e intercambio de ideas, necesidades y aspiraciones, constituyendo el sitio adecuado donde impulsar la búsqueda de igualdad (Mangas, 2002). Por tanto, es muy pertinente y relevante conocer si las universidades con mayor proporción de uno de los sexos tienen mejores niveles de producción científica, de internacionalización, de reputación, de relación con las empresas, en suma, mejor posición en los rankings universitarios internacionales.

\section{METODOLOGÍA}

En primer lugar, se han recopilado los rankings universitarios con los que trabajar, que son los siguientes:

- Academic Ranking of World Universities (ARWU): http://www.shanghairanking.com

- Center for World University Rankings (CWUR): https://cwur.org/

- National Taiwan University (NTU): http://nturanking.lis.ntu.edu.tw/

- Quacquarelli Symonds-QS ranking (QS): http://www.topuniversities.com/universityrankings

- Scimago Institutions Rankings (SCIMAGO): https://www.scimagojr.com/

- Times Higher Education (THE): https://www. timeshighereducation.com/world-universityrankings

- University Ranking by Academic Performance (URAP): http://www.urapcenter.org

Los seleccionados cumplen los siguientes requisitos: son rankings globales (abarcan universidades de todo el mundo), sintéticos porque dan una puntuación resumen (Luque-Martínez, Faraoni y Doña, 2018) y proporcionan información detallada. No se utilizaron otros rankings, como por ejemplo el ranking de Leiden, porque se decidió utilizar solo rankings sintéticos o que dieran una puntuación-resumen de los indicadores que utilizan. Estos rankings son los detallados en la tabla I que informa sobre el número de universidades que considera cada ranking y los criterios con los que se elabora cada uno de ellos (los criterios de elaboración se pueden ver en la respectiva web y en el anexo). Son rankings conocidos y muy utilizados en la literatura sobre clasificaciones académicas. De todos ellos se tiene la puntuación global y la posición de cada universidad, y la puntuación en cada uno de los criterios que aplican (a excepción del caso de SCIMAGO). Los datos se obtienen de las webs oficiales de cada ranking. 
Tabla I. Rankings universitarios seleccionados para el análisis

\begin{tabular}{|l|l|c|l|}
\hline \multicolumn{1}{|c|}{ Ranking } & \multicolumn{1}{c|}{ Denominación } & No de universidades & \multicolumn{1}{c|}{ Criterios } \\
\hline A: ARWU & $\begin{array}{l}\text { Academic Ranking of } \\
\text { World Universities }\end{array}$ & 500 & Alumni, Award, HiCi, N\&S, PUB., PCP. \\
\hline C: CWUR & $\begin{array}{l}\text { Center for World } \\
\text { University Rankings }\end{array}$ & 1000 & $\begin{array}{l}\text { Quality Education, Alumni Employment, } \\
\text { Quality of Faculty, Publications, Influence, } \\
\text { Citations, Broad Impact, Patents }\end{array}$ \\
\hline N: NTU & $\begin{array}{l}\text { Performance Ranking } \\
\text { of Scientific Papers for } \\
\text { World Universities }\end{array}$ & 500 & $\begin{array}{l}\text { 11 Years Articles, Current Articles, 11 Years } \\
\text { Citations, Current Citations, Ave. Citation, } \\
\text { H-Index, HiCi Papers, Hi-Impact Journal } \\
\text { Articles }\end{array}$ \\
\hline Q: QS & $\begin{array}{l}\text { Quacquarelli Symonds } \\
\text { Ranking }\end{array}$ & 936 & $\begin{array}{l}\text { Academic Reputation, Employer Reputation, } \\
\text { Faculty Student, Citation per Faculty, } \\
\text { International }\end{array}$ \\
\hline S: SCIMAGO & SCIMAGO & 2894 & Position \\
\hline T: THE & Times Higher Education & 980 & $\begin{array}{l}\text { Teaching, Research, Citation, } \\
\text { Internationalization, Industry Income }\end{array}$ \\
\hline U: URAP & $\begin{array}{l}\text { University Ranking by } \\
\text { Academic Performance }\end{array}$ & 2000 & $\begin{array}{l}\text { Article, Citation, Total Document, AIT, CIT, } \\
\text { Collaboration }\end{array}$ \\
\hline
\end{tabular}

$\mathrm{HiCi}$ : Investigadores con alto índice de citación en diversas materias. / N\&S: Artículos publicados en Nature y Science. / PUB: Artículos indexados en Science Citation Index - Expanded y Social Science Citation Index. / PCP: Rendimiento académico per cápita de una institución. / AIT: Impacto total de los artículos. / CIT: Impacto total de citas.

Posteriormente, se ha elaborado la base de datos conjunta con todos los rankings a 31 de diciembre de 2017. Esto implica un gran esfuerzo de recogida y depuración de datos, con una revisión de todas las denominaciones de las universidades en cada ranking, descubriendo y aclarando posibles discordancias entre un ranking y otro, por las diferentes denominaciones con las que aparecen las universidades.

La variable "porcentaje de mujeres dentro del alumnado" en la universidad, proporcionada por el ranking $\mathrm{THE}$, se dispone para 924 universidades, es el dato que se toma como referencia. Esta variable se ha recodificado en tres categorías correspondientes a los tres terciles y, por tanto, con una frecuencia similar en cada categoría. La primera categoría (1) comprende las universidades con mayor porcentaje de hombres (solamente un $47 \%$ o menos eran mujeres), la segunda categoría (2) incluye las universidades con un reparto equilibrado por género (más del $47 \%$ y menos del $55 \%$ eran mujeres), y la tercera categoría (3) agrupa a las universidades con más porcentaje de mujeres entre el alumnado ( $55 \%$ o más del total eran mujeres).

Finalmente, para el análisis de datos se aplican correlaciones de rangos, correlación de Pearson, ANOVA, y test no paramétricos mediante el software SPSS Statistics v20.

\section{ANÁLISIS DE DATOS}

La distinción en tres categorías de universidades (baja presencia de mujeres o presencia mayoritaria de hombres, equilibrio entre hombres y mujeres y porcentaje mayoritario de mujeres) presenta diferencias significativas en casi todas las situaciones.

Para el ranking URAP (tabla II), elaborado con indicadores bibliométricos que miden la producción de artículos, las citas, la productividad científica (AIT), el impacto total de las citas (CIT) o las publicaciones con colaboración internacional (COLLABORATION), en todos los indicadores hay diferencias estadísticamente significativas, correspondiendo los valores más altos a las universidades de la segunda categoría, es decir, las que presentan una estructura equilibrada por sexo.

El ranking NTU (tabla II) se elabora también con indicadores bibliométricos, la mayoría de los cuales consideran un período de 11 años. Tanto en los referidos al año precedente (número de artículos, número de citas, índice $\mathrm{H}$, artículos en revistas de alto impacto), como en los referidos al período de 11 años (artículos, citas, medida de citas, artículos altamente citados), hay diferencias estadísticamente significativas. El tipo de universidades que presentan los valores más altos es el segundo, es decir, las universidades que tienen una composición equilibrada por sexo. 
Tabla II. Valoraciones medias de los indicadores de los rankings URAP y NTU según distribución por género de la universidad (ANOVA)

\begin{tabular}{|c|c|c|c|c|c|c|c|}
\hline $\begin{array}{c}\text { Indicadores Ran- } \\
\text { king URAP }\end{array}$ & $\begin{array}{c}\text { \% de muje- } \\
\text { res alumnas } \\
\text { según la } \\
\text { categoría } \\
\text { (baja 1; } \\
\text { media 2; } \\
\text { alta 3) }\end{array}$ & $\begin{array}{c}\text { Número de } \\
\text { universida- } \\
\text { des }\end{array}$ & $\begin{array}{l}\text { Media re- } \\
\text { sultado del } \\
\text { análisis } \\
\text { ANOVA }\end{array}$ & $\begin{array}{l}\text { Indicadores } \\
\text { Ranking NTU }\end{array}$ & $\begin{array}{c}\text { \% de muje- } \\
\text { res alumnas } \\
\text { según la ca- } \\
\text { tegoría (baja } \\
\text { 1; media 2; } \\
\text { alta 3) }\end{array}$ & $\begin{array}{l}\text { Número de } \\
\text { universida- } \\
\text { des }\end{array}$ & $\begin{array}{l}\text { Media re- } \\
\text { sultado del } \\
\text { análisis } \\
\text { ANOVA }\end{array}$ \\
\hline \multirow{4}{*}{ ARTICULE * } & 1 & 286 & 65,55 & \multirow{4}{*}{$\begin{array}{l}11 \text { YEARS } \\
\text { ARTICLES * }\end{array}$} & 1 & 102 & 56,42 \\
\hline & 2 & 309 & 69,39 & & 2 & 167 & 57,27 \\
\hline & 3 & 287 & 64,48 & & 3 & 131 & 53,47 \\
\hline & Total & 882 & 66,55 & & Total & 400 & 55,81 \\
\hline \multirow{4}{*}{ CITATION * } & 1 & 286 & 63,92 & \multirow{4}{*}{$\begin{array}{l}\text { CURRENT } \\
\text { ARTICLES * }\end{array}$} & 1 & 102 & 56,98 \\
\hline & 2 & 309 & 67,58 & & 2 & 167 & 57,17 \\
\hline & 3 & 287 & 63,65 & & 3 & 131 & 53,27 \\
\hline & Total & 882 & 65,11 & & Total & 400 & 55,84 \\
\hline \multirow{4}{*}{$\begin{array}{c}\text { TOTAL } \\
\text { DOCUMENT * }\end{array}$} & 1 & 286 & 30,35 & \multirow{4}{*}{$\begin{array}{l}11 \text { YEARS } \\
\text { CITATIONS ** }\end{array}$} & 1 & 102 & 54,2 \\
\hline & 2 & 309 & 32,34 & & 2 & 167 & 56,3 \\
\hline & 3 & 287 & 30,62 & & 3 & 131 & 53,13 \\
\hline & Total & 882 & 31,14 & & Total & 400 & 54,73 \\
\hline \multirow{4}{*}{ AIT * } & 1 & 286 & 55,41 & \multirow{4}{*}{$\begin{array}{c}\text { CURRENT } \\
\text { CITATIONS ** }\end{array}$} & 1 & 102 & 55,47 \\
\hline & 2 & 309 & 58,61 & & 2 & 167 & 56,79 \\
\hline & 3 & 287 & 55,4 & & 3 & 131 & 53,76 \\
\hline & Total & 882 & 56,53 & & Total & 400 & 55,46 \\
\hline \multirow{4}{*}{ CIT ** } & 1 & 286 & 43,14 & \multirow{4}{*}{$\begin{array}{l}\text { AVE. } \\
\text { CITATIONS * }\end{array}$} & 1 & 102 & 51,63 \\
\hline & 2 & 309 & 45,15 & & 2 & 167 & 55,65 \\
\hline & 3 & 287 & 43,21 & & 3 & 131 & 55,07 \\
\hline & Total & 882 & 43,87 & & Total & 400 & 54,44 \\
\hline \multirow{4}{*}{ COLLABORATION * } & 1 & 286 & 45,76 & \multirow{4}{*}{$\mathrm{H}$-INDEX $* * *$} & 1 & 102 & 56,01 \\
\hline & 2 & 309 & 49,41 & & 2 & 167 & 58,15 \\
\hline & 3 & 287 & 47,42 & & 3 & 131 & 56,06 \\
\hline & Total & 882 & 47,58 & & Total & 400 & 56,92 \\
\hline \multirow{4}{*}{ SCORE $*$} & 1 & 286 & 304,13 & \multirow{4}{*}{$\begin{array}{l}\text { HICI PAPERS } \\
* *\end{array}$} & 1 & 102 & 53,97 \\
\hline & 2 & 309 & 322,49 & & 2 & 167 & 55,98 \\
\hline & 3 & 287 & 304,78 & & 3 & 131 & 52,79 \\
\hline & Total & 882 & 310,78 & & Total & 400 & 54,42 \\
\hline \multirow{8}{*}{ POSITION * } & 1 & 286 & 715,42 & \multirow{4}{*}{$\begin{array}{l}\text { HI-IMPACT } \\
\text { JOURNAL } \\
\text { ARTICLES ** }\end{array}$} & 1 & 102 & 55,13 \\
\hline & 2 & 309 & 565,29 & & 2 & 168 & 56,08 \\
\hline & 3 & 287 & 681,62 & & 3 & 131 & 52,71 \\
\hline & Total & 882 & 651,82 & & Total & 401 & 54,74 \\
\hline & & & & \multirow{4}{*}{ SCORE $* *$} & 1 & 102 & 55 \\
\hline & & & & & 2 & 168 & 56,33 \\
\hline & & & & & 3 & 131 & 53,62 \\
\hline & & & & & Total & 401 & 55,11 \\
\hline & & & & \multirow{4}{*}{ POSITION ** } & 1 & 102 & 252,74 \\
\hline & & & & & 2 & 167 & 215,62 \\
\hline & & & & & 3 & 131 & 266,85 \\
\hline & & & & & Total & 400 & 241,86 \\
\hline
\end{tabular}

(*) significación $1 \%$. (**) significación 5\%. (***) significación 10\%. (ns): no significativo.

AIT: Impacto total de los artículos / CIT: Impacto total de citas. 
En todos los indicadores del ranking ARWU (tabla III) hay diferencias estadísticamente significativas. En los indicadores de naturaleza claramente bibliométrica, como los autores altamente citados (HiCi), las publicaciones en Nature y Science (N\&S), o los artículos publicados (PUB), las universidades que alcanzan mayor puntuación son, de nuevo, las universidades que presentan un mayor equilibrio entre hombres y mujeres. Mientras que para los indicadores que tienen que ver con alumnos (ALUMNI), o profesores (AWARD) que consiguieron un premio Nobel o unas medallas Fields, las universidades que alcanzan mayor puntuación media son las de la categoría primera, las de mayor porcentaje de hombres. Recuérdese que son los hombres los que acaparan este tipo de premios. En ese mismo sentido, hay diferencia significativa para la producción científica según el número de profesores a tiempo completo (PCP).

El único indicador del ranking THE (tabla III) que no presenta diferencias estadísticamente significativas, según la distribución por género de las universidades, es el número de estudiantes equivalentes a tiempo completo (FTE) (se considera a tiempo completo aquel alumno matriculado en el total de créditos previstos por el curso, es decir, un alumno a tiempo completo es aquel que realiza 60 créditos ECTS. Si solo cursa 30 créditos ECTS equivale a un 0,5 del tiempo completo). En todos los demás casos, hay diferencias estadísticamente significativas siendo, otra vez, las universidades con una composición más equilibrada entre hombres y mujeres las que alcanzan mayor puntuación media. Esto ocurre con los indicadores de internacionalización (INTERNATIONAL), con los que miden la reputación de la enseñanza (TEACHING), o de la investigación (RESEARCH) y su nivel de visibilidad (CITATIONS). Sin embargo, son las universidades mayoritariamente compuestas por hombres las que tienen mayores ingresos procedentes de contratos con la industria. Seguramente, esto tiene que ver con que las universidades más técnicas son las que tienen más relaciones con la industria $y$, precisamente, son las que tienen las titulaciones con menor presencia de mujeres. Por el contrario, las universidades más masificadas, mayor ratio número de estudiantes por profesor, son las universidades con mayor porcentaje de mujeres alumnas.

En el caso de los indicadores del ranking QS (tabla IV), según el porcentaje de mujeres dentro del alumnado, las universidades no presentan diferencias estadísticamente significativas para la reputación académica. Sí hay diferencias estadísticamente significativas con mayor puntuación para las universidades con mayor porcentaje de hombres para los indicadores de reputación de los empleados (EMPLOYER REPUTATION), citas por autor, y ratio profesor por estudiante (FACULTY STUDENT). También hay dife- rencias significativas, con mayor puntuación para las universidades con composición más equilibrada por sexo, para los indicadores de internacionalización.

El ranking CWUR (tabla IV) no presenta diferencias estadísticamente significativas para su indicador de calidad de educación. Sí, hay diferencias, con mayor puntuación media, para las universidades con mayor porcentaje de hombres para el número de publicaciones, citas, e influencia de las citas (INFLUENCE). Asimismo, las universidades que tienen mayor porcentaje de mujeres estudiantes presentan mayores valores para la ratio del número de egresados que han conseguido puestos relevantes en grandes compañías según el tamaño de la universidad (ALUMNI EMPLOYMENT), calidad del profesorado (QUALITY OF FACULTY) y número de patentes.

Es de destacar que para todos los rankings hay diferencias estadísticamente significativas en la puntuación media que alcanzan y en la posición. En todos los casos, la puntuación media mayor $y$, por tanto, la posición mejor (la más baja) la consigue el grupo de universidades con una composición más equilibrada entre hombres y mujeres.

Con una total significación estadística, las universidades con menor porcentaje de mujeres alumnas se encuentran en los países con mayor población en términos absolutos, que también son los que tienen más población femenina. En esto, tiene que ver la situación de países muy poblados en Asia (China, India, Japón) o América Latina (como Brasil) caracterizados por universidades con reducida presencia femenina.

Por otro lado, las universidades que se encuentran en territorios con el PIB per cápita mayor son las que tienen un reparto equilibrado entre hombres y mujeres, con diferencias estadísticamente significativas.

Con respecto a la tabla de correlaciones (tabla $\mathrm{V})$, entre los indicadores con los que se elaboran estos rankings, la presencia de mujeres está correlacionada positivamente, aunque débilmente, con:

- Indicadores relativos a la internacionalización, como es el de colaboración internacional de URAP, o los relativos al nivel de internacionalización general, o por el número de estudiantes internacionales, como los indicadores de THE:

- El número de citas medias y totales de los autores de la universidad, como muestran los indicadores de NTU y THE;

- La ratio de estudiantes por profesor;

- La ratio de número de egresados que han conseguido puestos relevantes en grandes compañías, según el tamaño de la universidad (CWUR);

- El número de patentes de la universidad (CWUR). 
Tabla III. Valoraciones medias de los indicadores de los rankings ARWU y THE según distribución por género de la universidad (ANOVA)

\begin{tabular}{|c|c|c|c|c|c|c|c|}
\hline $\begin{array}{c}\text { Indicadores Ran- } \\
\text { king ARWU }\end{array}$ & $\begin{array}{l}\text { \% de muje- } \\
\text { res alumnas } \\
\text { según la } \\
\text { categoría } \\
\text { (baja 1; } \\
\text { media 2; } \\
\text { alta 3) }\end{array}$ & $\begin{array}{c}\text { Número de } \\
\text { universida- } \\
\text { des }\end{array}$ & $\begin{array}{l}\text { Media re- } \\
\text { sultado del } \\
\text { análisis } \\
\text { ANOVA }\end{array}$ & $\begin{array}{l}\text { Indicadores } \\
\text { Ranking THE }\end{array}$ & $\begin{array}{l}\text { \% de muje- } \\
\text { res alumnas } \\
\text { según la } \\
\text { categoría } \\
\text { (baja 1; } \\
\text { media 2; } \\
\text { alta 3) }\end{array}$ & $\begin{array}{c}\text { Número de } \\
\text { universida- } \\
\text { des }\end{array}$ & $\begin{array}{l}\text { Media re- } \\
\text { sultado del } \\
\text { análisis } \\
\text { ANOVA }\end{array}$ \\
\hline \multirow{4}{*}{ ALUMNI $* * *$} & 1 & 111 & 10,22 & \multirow{4}{*}{ TEACHING * } & 1 & 308 & 29,93 \\
\hline & 2 & 169 & 9,02 & & 2 & 316 & 32,18 \\
\hline & 3 & 124 & 6,65 & & 3 & 294 & 26,70 \\
\hline & Total & 404 & 8,62 & & Total & 918 & 29,67 \\
\hline \multirow{4}{*}{ AWARD * } & 1 & 111 & 10,41 & \multirow{4}{*}{$\begin{array}{c}\text { INTERNATIONAL } \\
\text { OUTLOOK * }\end{array}$} & 1 & 308 & 38,57 \\
\hline & 2 & 169 & 8,11 & & 2 & 316 & 52,52 \\
\hline & 3 & 124 & 4,20 & & 3 & 294 & 52,15 \\
\hline & Total & 404 & 7,54 & & Total & 918 & 47,72 \\
\hline \multirow{4}{*}{$\mathrm{HiCi} *$} & 1 & 111 & 15,96 & \multirow{4}{*}{ RESEARCH $*$} & 1 & 308 & 24,81 \\
\hline & 2 & 169 & 18,55 & & 2 & 316 & 28,81 \\
\hline & 3 & 124 & 13,95 & & 3 & 294 & 22,11 \\
\hline & Total & 404 & 16,43 & & Total & 918 & 25,32 \\
\hline \multirow{4}{*}{$\mathrm{N} \& \mathrm{~S} * *$} & 1 & 111 & 16,17 & \multirow{4}{*}{ CITATIONS * } & 1 & 308 & 40,23 \\
\hline & 2 & 168 & 16,90 & & 2 & 316 & 55,71 \\
\hline & 3 & 124 & 13,43 & & 3 & 294 & 51,53 \\
\hline & Total & 403 & 15,63 & & Total & 918 & 49,18 \\
\hline \multirow{4}{*}{ PUB * } & 1 & 111 & 39,59 & \multirow{4}{*}{$\begin{array}{c}\text { INDUSTRY INCO- } \\
\text { ME * }\end{array}$} & 1 & 308 & 50,91 \\
\hline & 2 & 169 & 42,30 & & 2 & 316 & 44,48 \\
\hline & 3 & 124 & 38,00 & & 3 & 294 & 40,29 \\
\hline & Total & 404 & 40,24 & & Total & 918 & 45,30 \\
\hline \multirow{4}{*}{ PCP ** } & 1 & 111 & 25,33 & \multirow{4}{*}{$\begin{array}{l}\text { NO. OF FTE STU- } \\
\text { DENTS (ns) }\end{array}$} & 1 & 311 & 27,19 \\
\hline & 2 & 169 & 23,42 & & 2 & 318 & 25,90 \\
\hline & 3 & 124 & 22,54 & & 3 & 295 & 25,94 \\
\hline & Total & 404 & 23,67 & & Total & 924 & 26,35 \\
\hline \multirow{4}{*}{ SCORE * } & 1 & 111 & 19,98 & \multirow{4}{*}{$\begin{array}{c}\text { STUDENT/STAFF } \\
*\end{array}$} & 1 & 310 & 17,28 \\
\hline & 2 & 169 & 20,39 & & 2 & 318 & 19,34 \\
\hline & 3 & 124 & 16,84 & & 3 & 295 & 20,80 \\
\hline & Total & 404 & 19,19 & & Total & 923 & 19,11 \\
\hline \multirow{4}{*}{ POSITION * } & 1 & 111 & 251,86 & \multirow{4}{*}{$\begin{array}{l}\text { INTERNATIONAL } \\
\text { STUDENT * }\end{array}$} & 1 & 296 & 0,10 \\
\hline & 2 & 169 & 214,85 & & 2 & 304 & 0,15 \\
\hline & 3 & 124 & 272,05 & & 3 & 286 & 0,13 \\
\hline & Total & 404 & 242,57 & & Total & 886 & 0,13 \\
\hline & & & & \multirow{4}{*}{ SCORE * } & 1 & 311 & 32,68 \\
\hline & & & & & 2 & 318 & 40,07 \\
\hline & & & & & 3 & 295 & 35,04 \\
\hline & & & & & Total & 924 & 35,98 \\
\hline & & & & \multirow{4}{*}{ POSITION * } & 1 & 311 & 556,54 \\
\hline & & & & & 2 & 318 & 426,98 \\
\hline & & & & & 3 & 295 & 491,62 \\
\hline & & & & & Total & 924 & 491,22 \\
\hline
\end{tabular}

(*) significación $1 \%$. (**) significación 5\%. (***) significación $10 \%$. (ns): no significativo.

$\mathrm{HiCi}$ : Investigadores con alto índice de citación en diversas materias. / N\&S: Artículos publicados en Nature y Science. / PUB: Artículos indexados en Science Citation Index - Expanded y Social Science Citation Index. / PCP: Rendimiento académico per cápita de una institución. 
Tabla IV. Valoraciones medias de los indicadores de los rankings QS, CWUR y de posición SCIMAGO, según distribución por género de la universidad (ANOVA)

\begin{tabular}{|c|c|c|c|c|c|c|c|}
\hline $\begin{array}{c}\text { Indicador } \\
\text { Ranking QS }\end{array}$ & $\begin{array}{l}\text { \% de muje- } \\
\text { res alumnas } \\
\text { según la ca- } \\
\text { tegoría (baja } \\
\text { 1; media 2; } \\
\text { alta 3) }\end{array}$ & $\begin{array}{l}\text { Número } \\
\text { de uni- } \\
\text { versida- } \\
\text { des }\end{array}$ & $\begin{array}{l}\text { Media } \\
\text { resulta- } \\
\text { do del } \\
\text { análisis } \\
\text { ANOVA }\end{array}$ & $\begin{array}{c}\text { Indicador } \\
\text { Ranking CWUR }\end{array}$ & $\begin{array}{l}\text { \% de muje- } \\
\text { res alumnas } \\
\text { según la } \\
\text { categoría } \\
\text { (baja 1; } \\
\text { media 2; } \\
\text { alta } 3 \text { ) }\end{array}$ & $\begin{array}{l}\text { Número de } \\
\text { universida- } \\
\text { des }\end{array}$ & $\begin{array}{l}\text { Media re- } \\
\text { sultado del } \\
\text { análisis } \\
\text { ANOVA }\end{array}$ \\
\hline \multirow{4}{*}{$\begin{array}{c}\text { ACADEMIC } \\
\text { REPUTATION (ns) }\end{array}$} & 1 & 103 & 57,11 & \multirow{4}{*}{$\begin{array}{c}\text { QUALITY OF } \\
\text { EDUCATION (ns) }\end{array}$} & 1 & 92 & 182,42 \\
\hline & 2 & 142 & 56,25 & & 2 & 133 & 176,85 \\
\hline & 3 & 99 & 52,29 & & 3 & 81 & 197,67 \\
\hline & Total & 344 & 55,37 & & Total & 306 & 184,04 \\
\hline \multirow{4}{*}{$\begin{array}{c}\text { EMPLOYER } \\
\text { REPUTATION * }\end{array}$} & 1 & 113 & 63,76 & \multirow{4}{*}{$\begin{array}{c}\text { ALUMNI } \\
\text { EMPLOYMENT } * *\end{array}$} & 1 & 162 & 278,98 \\
\hline & 2 & 122 & 60,59 & & 2 & 195 & 274,41 \\
\hline & 3 & 77 & 53,12 & & 3 & 112 & 328,68 \\
\hline & Total & 312 & 59,90 & & Total & 469 & 288,95 \\
\hline \multirow{4}{*}{$\begin{array}{l}\text { FACULTY } \\
\text { STUDENT ** }\end{array}$} & 1 & 131 & 61,21 & \multirow{4}{*}{$\begin{array}{l}\text { QUALITY OF } \\
\text { FACULTY *** }\end{array}$} & 1 & 50 & 101,22 \\
\hline & 2 & 144 & 54,74 & & 2 & 88 & 114,59 \\
\hline & 3 & 99 & 55,23 & & 3 & 54 & 131,61 \\
\hline & Total & 374 & 57,14 & & Total & 192 & 115,90 \\
\hline \multirow{4}{*}{$\begin{array}{l}\text { CITATION PER } \\
\text { FACULTY * }\end{array}$} & 1 & 135 & 61,93 & \multirow{4}{*}{ PUBLICATIONS * } & 1 & 219 & 482,34 \\
\hline & 2 & 184 & 50,02 & & 2 & 248 & 371,11 \\
\hline & 3 & 105 & 45,02 & & 3 & 214 & 451,20 \\
\hline & Total & 424 & 52,57 & & Total & 681 & 432,05 \\
\hline \multirow{4}{*}{$\begin{array}{l}\text { INTERNATIONAL } \\
\text { FACULTY ** }\end{array}$} & 1 & 104 & 59,25 & \multirow{4}{*}{ INFLUENCE * } & 1 & 216 & 538,15 \\
\hline & 2 & 171 & 66,86 & & 2 & 248 & 377,19 \\
\hline & 3 & 133 & 60,76 & & 3 & 214 & 430,27 \\
\hline & Total & 408 & 62,93 & & Total & 678 & 445,22 \\
\hline \multirow{4}{*}{$\begin{array}{l}\text { INTERNATIONAL } \\
\text { STUDENTS (ns) }\end{array}$} & 1 & 107 & 57,77 & \multirow{4}{*}{ CITATIONS * } & 1 & 185 & 386,65 \\
\hline & 2 & 170 & 62,53 & & 2 & 226 & 302,74 \\
\hline & 3 & 128 & 58,97 & & 3 & 187 & 352,19 \\
\hline & Total & 405 & 60,15 & & Total & 598 & 344,16 \\
\hline \multirow{4}{*}{ SCORE $*$} & 1 & 205 & 33,98 & \multirow{4}{*}{ BROAD IMPACT * } & 1 & 219 & 508,36 \\
\hline & 2 & 246 & 34,37 & & 2 & 248 & 375,08 \\
\hline & 3 & 194 & 27,32 & & 3 & 214 & 439,57 \\
\hline & Total & 645 & 32,13 & & Total & 681 & 438,21 \\
\hline \multirow{4}{*}{ POSITION * } & 1 & 213 & 398,83 & \multirow{4}{*}{ PATENTS * } & 1 & 200 & 383,76 \\
\hline & 2 & 257 & 393,23 & & 2 & 229 & 358,79 \\
\hline & 3 & 218 & 488,89 & & 3 & 198 & 516,28 \\
\hline & Total & 688 & 425,28 & & Total & 627 & 416,48 \\
\hline & & & & \multirow{4}{*}{ SCORE * } & 1 & 219 & 47,97 \\
\hline & & & & & 2 & 248 & 48,12 \\
\hline & & & & & 3 & 214 & 46,26 \\
\hline & & & & & Total & 681 & 47,49 \\
\hline \multirow{4}{*}{$\begin{array}{l}\text { SCIMAGO } \\
\text { POSITION * }\end{array}$} & 1 & 302 & 441,32 & \multirow{4}{*}{ POSITION * } & 1 & 219 & 476,07 \\
\hline & 2 & 314 & 395,32 & & 2 & 248 & 379,00 \\
\hline & 3 & 290 & 452,43 & & 3 & 214 & 469,45 \\
\hline & Total & 906 & 428,94 & & Total & 681 & 438,64 \\
\hline
\end{tabular}

(*) significación $1 \% .\left(^{* *}\right)$ significación $5 \% .(* * *)$ significación 10\%. (ns): no significativo. 
Tabla V. Correlaciones entre el porcentaje de mujeres dentro del alumnado en la universidad y los indicadores de los rankings ARWU, NTU, URAP, THE, QS y CWUR

\begin{tabular}{|l|l|c|c|}
\hline \multicolumn{1}{|c|}{ Ranking } & \multicolumn{1}{|c|}{ Indicador } & Correlación + & Significación \\
\hline URAP & COLLABORATION & 0,099 & 0,003 \\
\hline NTU & AVE.CITATIONS & 0,169 & 0,001 \\
\hline THE & INTERNATIONAL OUTLOOK & 0,254 & 0,000 \\
\hline THE & CITATIONS & 0,178 & 0,000 \\
\hline THE & STUDENT STAFF & 0,132 & 0,000 \\
\hline THE & INTERNATIONAL STUDEN & 0,114 & 0,000 \\
\hline CWUR & ALUMNI EMPLOYMENT & 0,098 & 0,033 \\
\hline CWUR & PATENTS Indicador & 0,175 & 0,000 \\
\hline & & Correlación - & Significación \\
\hline NTU & CURRENT ARTICLES & -0.103 & 0,040 \\
\hline THE & TEACHING & $-0,066$ & 0,046 \\
\hline THE & INDUSTRY INCOME & $-0,289$ & 0,000 \\
\hline QS & EMPLOYER REPUTATION & $-0,149$ & 0,008 \\
\hline QS & FACULTY ESTUDENT & $-0,148$ & 0,004 \\
\hline QS & CITATION PER FACULTY & $-0,277$ & 0,000 \\
\hline CWUR & PUBLICATIONS & $-0,076$ & 0,046 \\
\hline CWUR & INFLUENCE & $-0,202$ & 0,000 \\
\hline CWUR & CITATIONS & $-0,081$ & 0,049 \\
\hline CWUR & BROAD IMPACT & $-0,137$ & 0,000 \\
\hline
\end{tabular}

Por el contrario, la presencia de mujeres en la universidad tiene una correlación negativa, también débilmente, con:

- El número de artículos de investigación publicados, según NTU y CWUR;

- Los indicadores de docencia, como la reputación en docencia y de los empleadores, según THE y QS, o la ratio de profesores/estudiantes según QS;

- Los ingresos provenientes de la industria, o el impacto de las publicaciones de la universidad (según THE y CWUR, respectivamente);

- Las citas por profesor, y la calidad e influencia de las citas (según QS y CWUR).

\section{CONCLUSIONES, RECOMENDACIONES, LIMITACIONES}

El talento es uno de los principales factores que fomentan el crecimiento y la competitividad de los países, de sus economías y de sus sistemas educativos. Asegurar el completo desarrollo de las competencias de toda (no de la mitad) la población, y del talento mundial es una necesidad para la so- ciedad futura. Cuando la mitad de la población no tiene las mismas posibilidades que la otra mitad, además de ser injusto, el mundo está perdiendo riqueza, ideas, habilidades y perspectivas.

No existen indicadores que puedan dar una imagen global de lo que supone la desigualdad de género en las instituciones de educación superior hoy en día; sin embargo, hay intentos de medir, evaluar, investigar e informar. El indicador utilizado en el ranking THE es uno de estos. Este trabajo ofrece la oportunidad para una reflexión y para empezar a implementar acciones dirigidas a la mayor participación femenina en el ámbito académico, y en particular en aquellas áreas donde, todavía, por una razón u otra, no se ha logrado la igualdad. Además, se ofrece una panorámica de las características demográficas y económicas de los países donde se ha logrado un mayor equilibrio, y que se pueden definir los más desarrollados socialmente.

En este contexto de necesidad de promoción de la igualdad de género, este trabajo analiza la presencia femenina en las universidades y su impacto en los rankings, algo no investigado hasta la fecha. De los resultados alcanzados, se pueden extraer algunas ideas y conclusiones de relevantes. 
En primer lugar, las universidades con una distribución más equilibrada por género tienen mejores puntuaciones y ocupan mejores posiciones en los rankings universitarios globales. Suelen estar en territorios con mayor PIB per cápita. De esto, se puede deducir que la presencia equilibrada de mujeres y hombres es una manifestación de desarrollo económico y social. Seguramente es este nivel de desarrollo socioeconómico lo que favorece esa composición equilibrada entre el alumnado. Sin embargo, sigue existiendo una gran diferencia en distribución por género de las universidades en países muy poblados, principalmente en Asia y América Latina.

En la mayoría de los indicadores de los rankings universitarios, las universidades con una composición equilibrada por sexo son las que alcanzan mayores puntuaciones medias. En el caso de indicadores de excelencia, como Premios Nobel y Medallas Fields, los hombres tienen una gran ventaja sobre las mujeres, y esto coincide con que en estos indicadores las universidades con mayor porcentaje de hombres son las que obtienen más puntuación. Como también sucede con el indicador de ingresos de las universidades procedentes de la industria, las universidades con mayor presencia de hombres tienen mayor puntuación media, hecho seguramente debido a que las universidades con mayor relación con la industria son las que tienen un mayor contenido técnico y de ingenierías, precisamente titulaciones en las que las mujeres son minoría, tal como reflejaba la mayoría de la literatura consultada. De alguna manera, se están reflejando esquemas sociales arraigados. Igualmente, la inercia desigualitaria existente se manifiesta en algunos indicadores de producción científica, citas por profesor, o influencia de las citas.

Las universidades con mayor presencia de mujeres son las más masificadas en términos relativos, es decir por número de estudiantes atendidos por profesor, y las que alcanzan mayores correlaciones en los indicadores de internacionalización. Se encuentra una relación entre apertura internacional y mayor presencia de mujeres en la universidad.

Con respecto a las colaboraciones internacionales, medidas a través del indicador "collaboration" URAP del ranking, las universidades que sacan mejores puntuaciones son aquellas que presentan un porcentaje equilibrado de mujeres y hombres estudiantes. Con respecto al desarrollo de la ca- rrera profesional, los resultados de otros trabajos indican que, en el contexto investigador, las colaboraciones de las mujeres suelen desarrollarse más a nivel nacional, mientras que los hombres se decantan hacia colaboraciones internacionales (Larivière y otros, 2013), en particular por lo que se refiere a los campos técnicos (Abramo y otros, 2019). Estos resultados pueden encontrar su explicación en que, aunque un reparto equilibrado siga presentando mejor desempeño universitario, las responsabilidades familiares que se atribuyen conscientemente o inconscientemente a las mujeres, y que ellas perciben, hacen que sean los hombres los que están más dispuestos a colaborar con académicos internacionales y a moverse geográficamente.

De todo ello se desprende la necesidad de promover la igualdad en los diferentes ámbitos universitarios. Conseguir esa igualdad es una manera de progresar socialmente. Promover los programas de intercambio internacional y de colaboración, además de ser una manera de influenciar positivamente los resultados investigadores, es una forma de favorecer la apertura y la participación de la mujer en la universidad (Abramo, 2019).

En esta línea debieran ir las políticas universitarias de los gobiernos de los sistemas universitarios y las decisiones de gestión de las propias universidades.

Como futuras líneas de investigación, sería interesante un análisis más pormenorizado por países, así como el análisis según la presencia de mujeres investigadoras o entre el profesorado, o en puestos directivos. En particular, un análisis de la presencia femenina en el profesorado, sería interesante relacionarla con volumen o calidad de la producción científica. Otra posible línea de investigación futura puede estar dirigida un análisis de la distribución por género que diferencie por área temáticas, algo que los rankings elegidos para este trabajo no hacen. Las limitaciones de este estudio son, principalmente, las propias de los rankings y los criterios seguidos para su elaboración. Sus puntuaciones y valoraciones hay que tratarlas con precaución, siendo conscientes de lo que miden. Además del hecho de que se ha tenido en cuenta la distribución por género del alumnado, y no de otros grupos relacionados con las universidades, lo que hay que tener presente en toda interpretación. 


\section{REFERENCIAS}

Abramo, G.; D'Angelo, C. A.; \& Di Costa, F. (2019). A gender analysis of top scientists' collaboration behavior: evidence from Italy. Scientometrics, 120, 405-418. https://doi.org/10.1007/s11192-019-03136-6

Aiston, S. J. (2011). Equality, justice and gender: Barriers to the ethical university for women. Ethics and Education, 6(3), 279-291. https://doi.org/10.1080/17 449642.2011.632721

Alba, A. (2000). La riqueza de las familias: mujer y mercado de trabajo en la España democrática. Ariel.

Arranz, F. (2001). Hombres y mujeres en el profesorado: un análisis de género. En García de León, M. A. y García de Cortázar, M. (eds.), Las académicas (Profesorado universitario y género), pp. 337-404. Madrid. Instituto de la Mujer.

Bird, S. R. (2011). Unsettling universities' incongruous, gendered bureaucratic structures: A case-study approach. Gender, Work \& Organization, 18(2), 202-230. https://doi.org/10.1111/j.1468-0432.2009.00510.x

Box-Steffensmeier, J. M.; Cunha, R. C.; Varbanov, R. A.; Hoh, Y. S. ; Knisley, M. L.; Holmes, M. A. (2015). Survival analysis of faculty retention and promotion in the social sciences by gender. PloS one, 10(11), e0143093. https://doi.org/10.1371/journal.pone.0143093

Braidotti, R. (1994). Nomadic subjects: Embodiment and sexual difference in contemporary feminist theory. New York: Columbia University Press.

Caputo, C.; Vargas, D.; Requena, J. (2016). Desvanecimiento de la brecha de género en la universidad venezolana. Interciencia, 41(3), 154-161

Carmona-Valdés, S. E. (2015). Hacia una educación con equidad. Praxis, 11(1), 8-18. https://doi. org/10.21676/23897856.1549

CE- Comisión Europea (2009). Comunicación de la comisión al consejo, al parlamento europeo y al comité económico y social europeo y al comité de las regiones. Igualdad entre mujeres y hombres. Bruselas. Disponible en: https://eur-lex.europa.eu/legal-content/ES/ ALL/?uri=CELEX:52009DC0077 [Fecha de consulta: febrero 2019].

Centro de Investigación y Documentación Educativa (CIDE) (1998). La presencia de las mujeres en el sistema educativo. Madrid: Instituto de la Mujer.

Conley, H.; Jenkins, S. (2011). Still 'a good job for a woman'? Women teachers' experiences of modernization in England and Wales. Gender, Work \& Organization, 18(5), 488-507. https://doi.org/10.1111/j.1468$0432.2011 .00573 . x$

Correll, S. J. (2001). Gender and the career choice process: The role of biased self-assessments. American Journal of Sociology, 106(6), 1691-1730. https://doi. org/10.1086/321299

Díez Gutiérrez, E. J.; Terrón Bañuelos, E.; Anguita Martínez, R. (2009). Percepción de las mujeres sobre el" te- cho de cristal". Revista interuniversitaria de formación del profesorado, 23(1), 27-40.

División de Estadística de la UNESCO (1996). Enseñanza Técnica y Profesional de nivel secundario: Participación femenina en los diferentes sectores de estudios en 1980 y 1992 . En: Clair, R. (ed.), La formación científica de las mujeres ¿Por qué hay tan pocas científicas?, pp. 33-50. Madrid, Unesco/La Catarata.

Docampo, D. (2008). Rankings internacionales y calidad de los sistemas universitarios. Revista de Educación, (1), 149-176.

Docampo, D. (2010). On using the Shanghai ranking to assess the investigation performance of university systems. Scientometrics, 86 (1), 77-92. https://doi. org/10.1007/s11192-010-0280-y

Duart, F. P.; Quiñones, A. O.; Tobarra, A. (1996). Las Demandas de titulaciones universitarias ante la reforma de los planes de estudio. Ministerio de Asuntos Sociales. Instituto de la mujer.

European Institute for Gender Equality. (2017). Gender Equality Index 2017 - Measuring gender equality in the European Union 2005-2015. UK: EIGE.

Gámez, E.; Marrero, H. (2003). Metas y motivos en la elección de la carrera universitaria: Un estudio comparativo entre psicología, derecho y biología. Anales de Psicología, 19(1), 121-131.

García, C. F. (2014). Desequilibrios de género en educación en la España Contemporánea: causas, indicadores y consecuencias. Áreas. Revista Internacional de Ciencias Sociales, (33), 49-60.

Gauchat, G.; Kelly, M.; Wallace, M. (2012). Occupational gender segregation, globalization, and gender earnings inequality in US metropolitan areas. Gender \& Society, 26(5), 718-747. https://doi. org/10.1177/0891243212453647

Giménez-Salinas, E. (2005). Mujeres en la Universidad: cien años de prohibiciones. En: Giménez-Salinas Colomer, E. (ed.), Doctas, Doctoras y Catedráticas. Cien años de acceso libre de la mujer a la universidad, pp. 5772. Disponible en: http://mujeresconciencia.com/app/ uploads/2015/02/Doctas_doctoras_Castellano_Completo.pdf

Grañeras, M. (2003). Las mujeres en los equipos directivos de los centros escolares en España. Organización y gestión educativa, 11 (3), 15-20.

Greenhaus, J.H.; Callanan, G.A. (1994). Career Management, 2nd ed, Dryden Press, Harcourt Brace College Publishers, Orlando, FL.

Grove, J. (2013). Gender leadership gap tackled by manifesto. Disponible en: https://www.timeshighereducation.com/news/gender-leadership-gap-tackled-bymanifesto/2002419.article

Hazelkorn, E. (2012). The Effects of Rankings on Student Choices and Institutional Selection. En Jongbloed, B.; 
Vossensteyn, H. (eds.), Access and Expansion PostMassification: Opportunities and Barriers to Further Growth in Higher Education Participation. London: Routledge.

Hernández Armenteros, J.; Pérez García, J. (2018). Demografía universitaria española: aproximación a su dimensión, estructura y evolución. Cuaderno de trabajo 9. Madrid: Studia XXI. Fundación Europea Sociedad y Educación.

Hoff Sommers, C. (2008). Why can't a woman be more like a man? The American, March-April, p. 12.

Howe-Walsh, L.; Turnbull, S. (2016). Barriers to women leaders in academia: tales from science and technology. Studies in Higher Education, 41(3), 415-428. https://doi.org/10.1080/03075079.2014.929102

Igbaria, M.; Wormley, W. (1992). Race effects on organizational experiences and career success among MIS managers and professionals. MIS Quarterly, 16(4), 507-529. https://doi.org/10.2307/249734

Igbaria, M.; Chidambaram, L. (1997). The impact of gender on career success of information systems professionals: A human-capital perspective. Information Technology \& People, 10(1), 63-86. https://doi. org/10.1108/09593849710166165

Ion, G.; Durán-Belloch, M. M.; Bernabeu-Tamayo, M. D. (2013). El profesorado y su percepción sobre la igualdad de género en la universidad. Revista Complutense de Educación, 24(1), 123-140. https://doi.org/10.5209/ rev_RCED.2013.v24.n1.41194

Larivière, V.; Ni, C.; Gingras, Y.; Cronin, B.; Sugimoto, C. R. (2013). Bibliometrics: Global gender disparities in science. Nature News, 504(7479), 211. https://doi. org/10.1038/504211a

Light, A.; Ureta, M. (1995). Early-career work experience and gender wage differentials. Journal of Labor Economics, 13(1), 121-154. https://doi.org/10.1086/298370

Lobel, S. A.; Clair, L. S. (1992). Effects of family responsibilities, gender, and career identity salience on performance outcomes. Academy of Management Journal, 35(5), 1057-1069. https://doi.org/10.5465/256540

López-Sáez, M. (1995) La elección de una carrera típicamente femenina o masculina. Desde una perspectiva psicosocial: la influencia del género. Madrid, Ministerio de Educación y Ciencia.

Lozano, F. A. (2004). Las mujeres y la universidad española: estructuras de dominación y disposiciones feminizadas en el profesorado universitario. Política $y$ sociedad, 41(2), 223-242.

Luque-Martínez, T.; Barrio-García, S. del (2016). Constructing a synthetic indicator of research activity. Scientometrics, 108 (3), 1049-1064. https://doi. org/10.1007/s11192-016-2037-8

Luque-Martínez, T.; Barrio, S. del; Sánchez, J.; Ibáñez, J. A.; Doña, L. (2014). Estudio de egresados de la Universidad de Granada. Editorial Universidad de Granada.
Luque-Martínez, T; Del Barrio, S.; Sánchez, J.; Ibáñez, J. A.; Doña, L. (2015). Estudio de egresados de la Universidad de Granada. Editorial Universidad de Granada.

Luque-Martínez, T.; Faraoni, N. (2020). Meta-ranking to position world universities. Studies in Higher Education, 45(4), 819-833. https://doi.org/10.1080/03075079.20 18.1564260

Luque-Martínez, T.; Faraoni, N.; Doña Toledo, L. (2018). Meta-ranking de universidades. Posicionamiento de las universidades españolas. Revista Española de Documentación Científica, 41(1), 1-16. https://doi. org/10.3989/redc.2018.1.1456

Mangas, M. D. L. O. G. (2002). Mujer y universidad: espacios de conocimiento y espacios de poder. Revista Historia de la Educación Latinoamericana, (4).

Martínez García, J. S. (2007). Clase social, género y desigualdad de oportunidades educativas. Revista de Educación, 342, 287-306.

Matus-Lopéz, M.; Gallego-Morón, N. (2015). Glass ceiling in the University. If I don't see it don't believe it. Revista Complutense de Educación, 26(3), 611-626. https:// doi.org/10.5209/rev_RCED.2015.v26.n3.44491

Mauleón, E.; Bordons, M. (2014). Indicadores de actividad tecnológica por género en España a través del estudio de patentes europeas. Revista Española de Documentación Científica, 37(2), e043. https://doi. org/10.3989/redc.2014.2.1093

McWhirter, E. H. (1997). Perceived barriers to education and career: Ethnic and gender differences. Journal of Vocational Behavior, 50(1), 124-140. https://doi. org/10.1006/jvbe.1995.1536

Ministerio de Ciencia, Innovación y Universidades (MCIU) (enero 2019). Anuario de Indicadores universitarios 2018.

Ministerio de Educación, Cultura y Deporte. (2016). Datos y cifras del sistema universitario español. Recuperado de http://www.educacionyfp.gob.es/servicios-alciudadano/estadisticas/universitaria/datos-cifras.html

Mora García de Lomas, J. M. (2017). Los estudiantes y la reputación de la universidad. Vida universitaria, Universidad de Navarra. Recuperado de https://www.unav.edu/ web/vida-universitaria/detalle-opinion2/2017/04/04/ los-estudiantes-y-la-reputaci \% C3\%B3n-de-launiversidad?articleId $=13417543$

Morley, L. (2014). Lost leaders: Women in the global academy. Higher Education Research \& Development, 33(1), 114-128. https://doi.org/10.1080/07294360.2 013.864611

Morrison, A. M.; White, R. P.; White, R. P.; Van Velsor, E. (1987). Breaking The Glass Ceiling: Can Women Reach The Top Of America's Largest corporations? Pearson Education.

Muñoz, M. T.; Mullet, E. (1990). Los determinantes de las preferencias profesionales de los adolescentes. Evaluación Psicológica, 6, 155-170. 
Navarro-Guzmán, C.; Casero-Martínez, A. (2012). Análisis de las diferencias de género en la elección de estudios universitarios. ESE. Estudios sobre educación, 22, 115-132.

Papadópulos, J.; Radakovich R. (2003). Estudio Comparado de Educación Superior y Género en América Latina $y$ el Caribe. IESALC-Unión de Universidades de América Latina. Recuperado http://www.ses.unam.mx/curso2013/pdf/Papadopulos2006.pdf

Pérez-Tyteca, P., Castro, E. (2011). La ansiedad Matemática y su red de influencias en la elección de carrera Universitaria. En Marín, M., Fernández, G., Blanco, L. J., Palarea, M. M. (Eds.), Investigación en Educación Matemática XV (pp. 471-480). Ciudad Real: Sociedad Española de Investigación en Educación Matemática, SEIEM.

Powell, G. N.; Butterfield, D. A.; Parent, J. D. (2002). Gender and managerial stereotypes: have the times changed? Journal of Management, 28(2), 177-193.

Sáinz, M.; López-Sáez, M.; Lisbona, A. (2004) Expectativas de rol profesional de mujeres estudiantes de carreras típicamente femeninas o masculinas. Acción Psicológica, 3, 111-123.

Seagram, B. C.; Gould, J.; Pyke, S. W. (1998). An investigation of gender and other variables on time to completion of doctoral degrees. Research in Higher Education, 39(3), 319-335. https://doi. org/10.1023/A: 1018781118312

Shavit, Y.; Blossfeld, H. P. (1996). Equalizing educational opportunity: Do gender and class compete? En Erikson, R. y O. Jonsson, J. (Eds.), Can education be equalized? The Swedish Case in Comparative Perspective, pp. 233-254. Boulder, Colorado: Westview Press.

Stroh, L.K.; Brett, J.M.; Riley, A.H. (1992). All the right stuff: a comparison of female and male managers' career progression. Journal of Applied Psychology, 77, 251-260. https://doi.org/10.1037/00219010.77.3.251

Sugimoto, C., Larivière, V. (2019). Indicators for social good. CWTS. Recuperado de https://www.cwts.nl/ blog?article $=n-r 2$ w2c4\&title $=$ indicators-for-socialgood

Summers, L. H. (2005). Remarks at NBER Conference on diversifying the science $\&$ engineering workforce Cambridge, MA.

Teelken, C.; Deem, R. (2013). All are equal, but some are more equal than others: Managerialism and gender equality in higher education in comparative perspective. Comparative Education, 49(4), 520-535. https://doi.org/10.1080/03050068.2013.807642

Tidball, M. E. (1976). Of men and research: The dominant themes in American higher education include neither teaching nor women. The Journal of Higher Education, 47(4), 373-389. https://doi.org/10.1080/00221546.1 976.11774062
Timmers, T. M.; Willemsen, T. M.; Tijdens, K. G. (2010). Gender diversity policies in universities: a multiperspective framework of policy measures. Higher Education, 59(6), 719-735. https://doi.org/10.1007/ s10734-009-9276-z

Trotter, L. J. (2017). Making A Career: Reproducing Gender within a Predominately Female Profession. Gender \& Society, 31(4), 503-525. https://doi. org/10.1177/0891243217716115

Tzanakou, C.; Pearce, R. (2019). Moderate feminism within or against the neoliberal university? The example of Athena SWAN. Gender, Work \& Organization, 26 (8), 1191, 1211. https://doi.org/10.1111/gwao.12336

UNESCO Institute for Statistics (2018). https://tellmaps. com/uis/gender/\#!/tellmap/79054752

Van den Brink, M.; Benschop, Y.; Jansen, W. (2010). Transparency in academic recruitment: a problematic tool for gender equality? Organization Studies, 31(11), 1459-1483.

Wagner, C. (15 de mayo de 2019). Measuring Inequality - Creating an indicator to assess gender bias in universities. LSE Impact Blog. Recuperado de https:// blogs. Ise.ac.uk/impactofsocialsciences/2019/05/15/ measuring-inequality-creating-an-indicator-to-assessgender-bias-in-universities/

Walby, S. (2011). Is the knowledge society gendered? Gender, Work and Organization, 18(1), 1-29. https:// doi.org/10.1111/j.1468-0432.2010.00532.x

Willemsen, T. M. (2002). Gender typing of the successful manager. A stereotype reconsidered. Sex Roles, 46, 385-391. https://doi.org/10.1023/A: 1020409429645

World Bank (2016). World Development Indicator. https://databank.worldbank.org/data/reports. as px ? source $=2$ \&series $=$ SE.PRM. TCHR. FE.ZS\&country=

World Economic Forum (2017). The Global Gender Gap Report 2017. Recuperado de http://www3.weforum. org/docs/WEF_GGGR_2017.pdf [Fecha de consulta: febrero 2019].

World Intellectual Property Organization (2016). Identifying the Gender of PCT inventors. Economics \& Statistics Series, 33. https://www.wipo.int/edocs/ pubdocs/en/wipo_pub_econstat_wp_33.pdf

Wright, T. (2016). Women's experience of workplace interactions in male-dominated work: The intersections of gender, sexuality and occupational group. Gender, work \& organization, 23(3), 348-362. https://doi. org/10.1111/gwao.12074

Zeegers, P. (2004). Student learning in higher education: A path analysis of academic achievement in science. Higher Education Research \& Development, 23(1), 3556. https://doi.org/10.1080/0729436032000168487 


\section{ANEXOS}

Anexo 1. Academic Ranking of World Universities, ARWU

\begin{tabular}{|l|l|c|}
\hline \multicolumn{1}{|c|}{ Criterio } & \multicolumn{1}{c|}{ Indicador } & Ponderación \\
\hline $\begin{array}{l}\text { Calidad de la Docencia } \\
\text { (Alumni) }\end{array}$ & $\begin{array}{l}\text { Antiguos alumnos de una institución con Premios Nobel y } \\
\text { Medallas Fields. }\end{array}$ & $10 \%$ \\
\hline $\begin{array}{l}\text { Calidad del Profesorado } \\
\text { (Award) }\end{array}$ & $\begin{array}{l}\text { Profesores de una institución que han obtenido Premios Nobel y } \\
\text { Medallas Fields. }\end{array}$ & $20 \%$ \\
\hline $\begin{array}{l}\text { (HiCi) } \\
\text { Investigadora (N\&S) }\end{array}$ & Anvestigadores con alto índice de citación en diversas materias. & $20 \%$ \\
\hline $\begin{array}{l}\text { (PUB) } \\
\text { Rendimiento per Cápita } \\
\text { (PCP) }\end{array}$ & $\begin{array}{l}\text { Artículos indexados en Science Citation Index - Expanded y Social } \\
\text { Science Citation Index. }\end{array}$ & $20 \%$ \\
\hline
\end{tabular}

Anexo 2. CWUR World University Rankings, CWUR

\begin{tabular}{|c|c|c|}
\hline Criterio & Indicador & Ponderación \\
\hline $\begin{array}{l}\text { Calidad de la educación } \\
\text { (Quality of Education) }\end{array}$ & $\begin{array}{l}\text { Se mide utilizando el número de alumnos de una universidad que han } \\
\text { ganado premios y reconocimientos internacionales, así como medallas } \\
\text { en relación al tamaño de la universidad. }\end{array}$ & $25 \%$ \\
\hline $\begin{array}{l}\text { Situación de egresados } \\
\text { (Alumni Employment) }\end{array}$ & $\begin{array}{l}\text { Basado en el número de estudiantes egresados que han conseguido } \\
\text { posiciones de mando en las principales multinacionales, en relación al } \\
\text { tamaño de la universidad. }\end{array}$ & $25 \%$ \\
\hline $\begin{array}{l}\text { Calidad de profesores } \\
\text { (Quality of Faculty) }\end{array}$ & $\begin{array}{l}\text { Se mide a través de la cantidad de profesores que han ganado un } \\
\text { mayor número de premios, reconocimientos internacionales y medallas } \\
\text { en relación al tamaño de la universidad. }\end{array}$ & $25 \%$ \\
\hline $\begin{array}{l}\text { Publicaciones (Research } \\
\text { Output) }\end{array}$ & Número de publicaciones en revistas de reconocido prestigio. & $5 \%$ \\
\hline Influencia (Influence) & Número de publicaciones en revistas de alta influencia. & $5 \%$ \\
\hline Citas (Citations) & Medido a través del número de artículos altamente citados. & $5 \%$ \\
\hline Impacto (Broad Impact) & Medido a través del índice h de la universidad. & $5 \%$ \\
\hline Patentes (Patents) & Número de patentes obtenidas. & $5 \%$ \\
\hline
\end{tabular}

Anexo 3. National Taiwan University Ranking, NTU

\begin{tabular}{|c|c|c|c|}
\hline Criterio & Indicador & \multicolumn{2}{|c|}{ Ponderación } \\
\hline \multirow{2}{*}{$\begin{array}{l}\text { Productividad } \\
\text { investigadora } \\
\text { (Research } \\
\text { productivity) }\end{array}$} & No de artículos en los últimos 11 años (2004-2014). & $10 \%$ & \multirow[t]{2}{*}{$25 \%$} \\
\hline & No de artículos en el año actual (2014). & $15 \%$ & \\
\hline \multirow{3}{*}{$\begin{array}{l}\text { Impacto de la } \\
\text { investigación } \\
\text { (Research } \\
\text { Impact) }\end{array}$} & No de citas en los últimos 11 años (2004-2014). & $15 \%$ & \multirow{3}{*}{$35 \%$} \\
\hline & No de citas en el año actual (2014). & $10 \%$ & \\
\hline & No medio de citas en los últimos 11 años (2004-2014). & $10 \%$ & \\
\hline \multirow{3}{*}{$\begin{array}{l}\text { Excelencia de } \\
\text { la investigación } \\
\text { (Research } \\
\text { Excellence) }\end{array}$} & Índice h de los dos últimos años (2013-14). & $10 \%$ & \multirow{3}{*}{$40 \%$} \\
\hline & No de artículos altamente citados (2004-2014). & $15 \%$ & \\
\hline & No de artículos en el año actual en revistas de alto impacto (2013-2014). & $15 \%$ & \\
\hline
\end{tabular}


Anexo 4. QS World University Rankings, QS

\begin{tabular}{|c|c|c|}
\hline Criterio & Indicador & Ponderación \\
\hline $\begin{array}{l}\text { Reputación académica } \\
\text { (Academic Reputation) }\end{array}$ & $\begin{array}{l}\text { Se mide utilizando una encuesta mundial en la que los académicos } \\
\text { tienen que identificar las instituciones donde creen que está teniendo } \\
\text { lugar el mejor trabajo dentro de su propio campo de experiencia. }\end{array}$ & $40 \%$ \\
\hline $\begin{array}{l}\text { Reputación de la } \\
\text { docencia (Employer } \\
\text { Reputation) }\end{array}$ & $\begin{array}{l}\text { Basado en una encuesta mundial contando con más de } 44.200 \\
\text { respuestas (para la edición 2015/16). La encuesta pide a los } \\
\text { empleadores de identificar las universidades que, según ellos, están } \\
\text { formando los mejores graduados. }\end{array}$ & $10 \%$ \\
\hline $\begin{array}{l}\text { Ratio alumnos/ } \\
\text { profesores (Faculty } \\
\text { Student) }\end{array}$ & Ratio entre profesorado y número de alumnos. & $20 \%$ \\
\hline $\begin{array}{l}\text { Citas por profesor } \\
\text { (Citation per Faculty) }\end{array}$ & $\begin{array}{l}\text { Midiendo el número de citas por profesor se intenta evaluar el impacto } \\
\text { de la investigación de la universidad. }\end{array}$ & $20 \%$ \\
\hline $\begin{array}{l}\text { Ratio estudiantes } \\
\text { extranjeros y ratio } \\
\text { profesores extranjeros } \\
\text { (International) }\end{array}$ & $\begin{array}{l}\text { Estos indicadores evalúan la capacidad de la institución de atraer } \\
\text { estudiantes y docentes extranjeros. }\end{array}$ & $5 \%+5 \%$ \\
\hline
\end{tabular}

Anexo 5. Times Higher Education, THE

\begin{tabular}{|c|c|c|}
\hline Criterio & Indicador & Ponderación \\
\hline Docencia (Teaching) & $\begin{array}{l}\text { Formado por encuestas de reputación, ratios estudiantes/profesorado, } \\
\text { ratio graduados/doctores e ingresos institucionales. }\end{array}$ & $30 \%$ \\
\hline Investigación (Research) & $\begin{array}{l}\text { Formado por encuestas de reputación, ingresos por investigación y } \\
\text { productividad de la investigación. }\end{array}$ & $30 \%$ \\
\hline Citas (Citation) & $\begin{array}{l}\text { Evalúa la influencia de una universidad midiendo el número de veces } \\
\text { que un trabajo se cita en una publicación, comparado con el número } \\
\text { de citas que se espera que tenga una publicación de tipo similar. }\end{array}$ & $30 \%$ \\
\hline $\begin{array}{l}\text { Visión internacional } \\
\text { (Internationalization) }\end{array}$ & $\begin{array}{l}\text { Formado por ratio estudiantes nacionales/internacionales, ratios } \\
\text { docentes nacionales/internacionales y colaboraciones internacionales } \\
\text { con otras universidades. }\end{array}$ & $7,5 \%$ \\
\hline $\begin{array}{l}\text { Ingresos de la industria } \\
\text { (Industry Income) }\end{array}$ & $\begin{array}{l}\text { Evalúa la actividad de transferencia de conocimientos midiendo los } \\
\text { ingresos de una institución procedentes de la industria, comparado con el } \\
\text { número de académicos que la investigación emplea. }\end{array}$ & $2,5 \%$ \\
\hline
\end{tabular}

Anexo 6. University Ranking by Academic Performance, URAP

\begin{tabular}{|l|l|c|}
\hline \multicolumn{1}{|c|}{ Criterio } & \multicolumn{1}{c|}{ Indicador } & Ponderación \\
\hline Artículos (Article) & $\begin{array}{l}\text { Artículos publicados en } 2013 \text { indexados en Web of Science y listados en } \\
\text { Incites. }\end{array}$ & $21 \%$ \\
\hline Citas (Citation) & $\begin{array}{l}\text { No de citas recibidas en los artículos publicados en 2011-2013 indexados } \\
\text { en la Web of Science. }\end{array}$ & 21\% \\
\hline $\begin{array}{l}\text { Documentos totales } \\
\text { (Total Document) }\end{array}$ & $\begin{array}{l}\text { Documentos totales (papers, reviews, letters, discussions, scripts) } \\
\text { además de los artículos publicados durante 2011-2013. }\end{array}$ & $10 \%$ \\
\hline $\begin{array}{l}\text { Impacto total de los } \\
\text { artículos (AIT) }\end{array}$ & $\begin{array}{l}\text { Medida de productividad científica normalizada por institución respecto a } \\
\text { la media mundial en 23 subáreas entre 2011-2013. }\end{array}$ & $18 \%$ \\
\hline $\begin{array}{l}\text { Impacto total de citas } \\
\text { (CIT) }\end{array}$ & $\begin{array}{l}\text { Medida de impacto científico normalizada por institución respecto a la } \\
\text { media mundial en } 23 \text { subáreas entre 2011-2013. }\end{array}$ & $15 \%$ \\
\hline $\begin{array}{l}\text { Colaboración } \\
\text { internacional } \\
\text { (Collaboration) }\end{array}$ & $\begin{array}{l}\text { Medida de la aceptación global de la universidad. No de publicaciones } \\
\text { realizadas con universidades extranjeras para 2011-2013. }\end{array}$ & $15 \%$ \\
\hline
\end{tabular}

